\title{
Pseudogap and Other Precursor Signatures in the Systems with Local Fermion Pairs
}

\author{
T. DOMAŃSKI \\ Institute of Physics, M. Curie Skłodowska University \\ pl. M. Curie-Skłodowskiej 1, 20-031 Lublin, Poland
}

\begin{abstract}
We consider precursor effects of the superconducting order which possibly show up in the normal state of high temperature superconducting materials. The local pairs of electrons or holes are formed there well above the transition temperature $T_{\mathrm{c}}$. Due to strong quantum fluctuations the single particle density of states can be partly depleted near the Fermi energy leading to the pseudogap. We claim that this feature should go hand in hand with emergence of the Bogoliubov-type quasiparticles. In the normal state they are expected to be damped and acquire the long lifetime upon passing $T_{\mathrm{c}}$. These Bogoliubov-type quasiparticles may nevertheless become correlated on a finite spatial and temporal scale. We discuss how such short-range pair correlations can be detected experimentally.
\end{abstract}

PACS numbers: 74.20.Mn, 71.10.Li, 03.75.Kk

\section{Precursor of superconductivity vs. competing order}

The high temperature superconductors (HTS) are electron or hole doped Mott insulators where pairing occurs between fermions (electrons or correspondingly holes) from the nearest or next nearest neighbor lattice sites. These local pairs are hence distinct from the Cooper pairs in classical superconductors. Important question which is presently under debate concerns the underlying mechanism responsible (a) for binding fermions into such local pairs, and (b) for a character of the phase transition when their long range coherence sets in. It seems that answer can be achieved from understanding of the pseudogap origin.

Pseudogap phase is present above $T_{\mathrm{c}}$ in the underdoped to optimally doped samples of HTS. First indirect indications came from measurements of the magnetic correlations revealing a spin gap in the neutron scattering as well as NMR 
relaxation rate and the Knight shift. Soon after also the charge transport properties exhibited the similar effect. The pseudogap which forms among the low lying states was unambiguously confirmed via the single particle measurements by angle-resolved photoemission spectroscopy (ARPES), scanning tunneling microscopy (STM), and the specific heat [1]. It came as a surprise that pseudogap has the same angular dependence (of $d_{x^{2}-y^{2}}$ symmetry) as the gap of superconducting state. The immediate and natural conclusion was to regard them as close relatives which reflect the common order parameter. This interpretation for pseudogap is presently termed as the precursor approach (see the left part of Fig. 1).
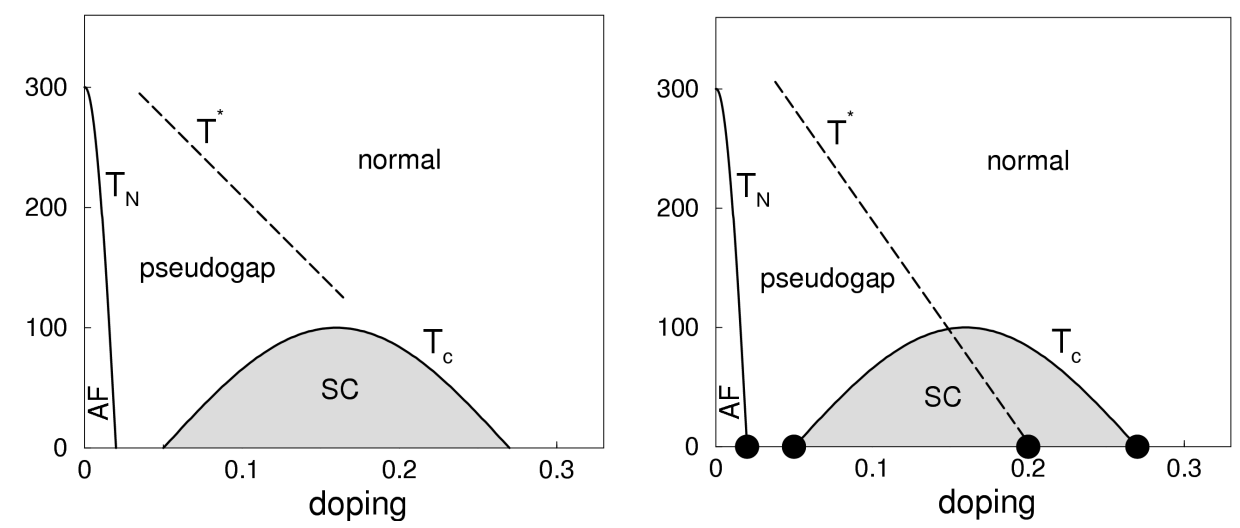

Fig. 1. Schematic view of the HTS phase diagram. Left part illustrates how the pseudogap is interpreted as a precursor of the superconducting order. The right part shows the interpretation in terms of the competing orders where possible quantum critical points are marked by the filled circles.

However, there exist also some conflicting experimental data which suggest that pseudogap and superconducting gap might be unrelated or that perhaps they correspond to different types of order competing with each other. Such conjecture is motivated e.g. by observation that the magnetic field and magnetic impurities (cobalt) influence both phases in distinct way [1]. Other stronger arguments were provided by ARPES with the use of the circularly polarized photons and by the recent STM experiments [2]. They revealed existence of microscopic currents (breaking the time reversal invariance) and charge density modulations (leading to "checkerboard" order). Among various theoretical speculations there is often emphasized a possible role of the quantum critical points (see the right part of Fig. 1) which correspond to transitions between different orders of the ground state upon doping.

At present none of these scenarios can be ruled out. In this work we would like to focus on arguments which are in favor for the precursor approach to pseudogap. It is obvious that precursor effects occur in the normal state of HTS cuprates $[3,4]$. There is only a controversy whether temperature region $T<T_{\mathrm{p}}$ of super- 
conducting fluctuations covers the whole pseudogap phase up to $T^{*}$ which by far exceeds $T_{\mathrm{c}}$. Independently of the final answer our discussion will remain valid for temperatures sufficiently close to the transition temperature $T_{\mathrm{c}}$ as recently judged by Uemura [5].

\section{Where and how one can recognize the precursor effects?}

In general, one expects strong influence of the superconducting order fluctuations when overlap of the fermion pair's wave functions is small. This is a case in the underdoped regime of HTS. The temperature $T_{\mathrm{p}}$, at which pairs are formed, and $T_{\mathrm{c}}$ when their long range coherence sets in should there differ considerably. In temperature region $T_{\mathrm{p}}>T>T_{\mathrm{c}}$ thus preformed pairs would be incoherent due to a combined effect of the phase and amplitude fluctuations.

In classical 3-dimensional superconductors fluctuations are usually restricted to a very narrow temperature regime around $T_{\mathrm{c}}$. One can theoretically describe them by considering the contribution of Aslamazov-Larkin [6] and MakiThompson diagrams to transport [7]. Superconducting order fluctuations were known experimentally in classical superconductors from measurements of the electrical conductivity [8], magnetic susceptibility [9], and single particle tunneling [10]. They all proved to be rather residual.

Fluctuations however start to play much stronger role in the low dimensional systems and/or when pairing occurs in the real space. Precursors of superconductivity can manifest themselves in the normal state by properties which are typical of the superconducting state. The most obvious of them is a reduction of the single particle states near the Fermi energy. Indirect consequences of its existence can be noticed further in the magnetic susceptibility, optical conductivity, etc. Besides these there might arise the many-body features such as e.g. the residual Meissner effect and the broadened Drude peak. They signify appearance of the fermion pairs correlated on some short-range distance.

\section{Phenomenological picture and the methodology}

One of possible routes for addressing the precursor effects is possible within the model describing a mixture of the single particle fermions and tightly bound pairs (hard-core bosons whose internal structure does not allow for double occupancy of the same lattice sites). Such boson fermion (BF) model has been introduced almost 20 years ago [11] and in connection with HTS was intensively explored by several groups [12]. Hamiltonian of the model is given by 


$$
\begin{aligned}
H= & \sum_{\boldsymbol{k}}, \sigma\left(\varepsilon_{\boldsymbol{k}}-\mu\right) c_{\boldsymbol{k} \sigma}^{\dagger} c_{\boldsymbol{k} \sigma}+\sum_{\boldsymbol{q}}\left(E_{\boldsymbol{q}}-2 \mu\right) b_{\boldsymbol{q}}^{\dagger} b_{\boldsymbol{q}} \\
& +\frac{1}{\sqrt{N}} \sum_{\boldsymbol{k}, \boldsymbol{q}} v_{\boldsymbol{k}, \boldsymbol{q}-\boldsymbol{k}}\left(c_{\boldsymbol{k} \uparrow}^{\dagger} c_{\boldsymbol{q}-\boldsymbol{k} \downarrow}^{\dagger} b_{\boldsymbol{q}}+\text { h.c. }\right),
\end{aligned}
$$

where $c_{\boldsymbol{k} \sigma}^{\dagger}\left(c_{\boldsymbol{k} \sigma}\right)$ describe the creation (annihilation) of single particle fermions (electrons or holes) with momentum $\boldsymbol{k}$ and spin $\sigma$ and operators $b_{\boldsymbol{q}}^{\dagger}\left(b_{\boldsymbol{q}}\right)$ refer correspondingly to the localized fermion pairs of arbitrary origin. To underline local character of the pairs we take a discrete energy for $E_{\boldsymbol{q}}$ (infinite mass). Correlations responsible for superconductivity are induced among fermions via the Andreev type scattering expressed in the last term of (1). For simplicity we will consider the isotropic coupling but in the real HTS materials it has the form $v_{\boldsymbol{k}, \boldsymbol{q}-\boldsymbol{k}}=v\left(\cos k_{x}-\cos k_{y}\right)$.

As usually $\mu$ denotes the chemical potential. If $2 \mu$ is close to $E_{\boldsymbol{q}=\mathbf{0}}$ then populations of fermions and bosons are comparable and consequently the transition temperature $T_{\mathrm{c}}$ can be very large. In such situation also the quantum fluctuations become pronounced and lead to a broad region $T_{\mathrm{p}}>T>T_{\mathrm{c}}$. In other cases the system consists predominantly of either single particle fermions (for $2 \mu \ll E_{\mathbf{0}}$ ) or bosonic particles (for $2 \mu>E_{\mathbf{0}}$ ). Correlations are then rather weak and lead correspondingly to the BCS type superconductivity of fermions or Bose-Einstein condensation (BEC) of bosons as discussed in the review paper [12].

In BF model superconducting correlations emerge gradually [13]: first there appear fermion pairs, later on they acquire longer and longer lifetime and finally fermion pairs establish the long-range coherence. This process results from the feedback effects induced by the boson fermion coupling $v_{\boldsymbol{k}, \boldsymbol{q}-\boldsymbol{k}}$. For studying the mutual relations between the single and two-particle properties one has to treat them on equal footing which is a very difficult task. In our study we use the renormalization group procedure [14] whose basic idea is to transform the initial Hamiltonian through a set of infinitesimal canonical transformations with a flexibly adjusted generating operator.

We have already designed such diagonalizing procedure in the previous paper [15] and extended it recently [16] for derivation of various dynamical quantities. Here we recapitulate the main aspects of this approach. During continuous transformation the quantities appearing in the Hamiltonian are renormalized according to the operator equation $\mathrm{d} H(l) / \mathrm{d} l=[\eta(l), H(l)]$, where $\eta$ is chosen after [14] as

$$
\eta(l)=\frac{1}{\sqrt{N}} \sum_{\boldsymbol{k}, \boldsymbol{p}} \alpha_{\boldsymbol{k}, \boldsymbol{p}}(l)\left(c_{\boldsymbol{p} \uparrow}^{\dagger} c_{\boldsymbol{k} \downarrow}^{\dagger} b_{\boldsymbol{p}+\boldsymbol{k}}-\text { h.c. }\right),
$$

where $\alpha_{\boldsymbol{k}, \boldsymbol{p}}(l)=\left[\varepsilon_{\boldsymbol{k}}(l)+\varepsilon_{\boldsymbol{p}}(l)-E_{\boldsymbol{k}+\boldsymbol{p}}(l)\right] v_{\boldsymbol{k}, \boldsymbol{p}}(l)$ [15]. Fermion and boson energies undergo renormalizations $\varepsilon_{\boldsymbol{k}} \rightarrow \varepsilon_{\boldsymbol{k}}(l), E_{\boldsymbol{q}} \rightarrow E_{\boldsymbol{q}}(l)$ until the coupling potential diminishes at $\lim _{l \rightarrow \infty} v_{\boldsymbol{k}, \boldsymbol{p}}(l)=0$. Besides renormalizing the Hamiltonian also the 
operators should be transformed in order to calculate the correlation functions. In the next sections we will briefly describe some of our results.

\section{Pair features in the single particle spectrum}

In the normal state the single particle fermion excitations are characterized by the following spectral function [16]:

$$
A^{\mathrm{F}}(\boldsymbol{k}, \omega)=\left|\tilde{\mathcal{P}}_{\boldsymbol{k}}\right|^{2} \delta\left(\omega-\tilde{\varepsilon}_{\boldsymbol{k}}\right)+\frac{1}{N} \sum_{\boldsymbol{q} \neq \mathbf{0}}\left(n_{\boldsymbol{q}}^{\mathrm{B}}+n_{\boldsymbol{q}-\boldsymbol{k} \downarrow}^{\mathrm{F}}\right)\left|\tilde{r}_{\boldsymbol{k}, \boldsymbol{q}}\right|^{2} \delta\left(\omega-\tilde{E}_{\boldsymbol{q}}+\tilde{\varepsilon}_{\boldsymbol{q}-\boldsymbol{k}}\right) .
$$

Coefficients $\tilde{\mathcal{P}}_{\boldsymbol{k}}, \tilde{r}_{\boldsymbol{k}, \boldsymbol{q}}$ are the convergence solutions (at $l \rightarrow \infty$ ) of the parameterization for fermion operators discussed at length in Ref. [16]. The single particle fermion spectrum is thus composed of the long-lived states at renormalized energies $\tilde{\varepsilon}_{\boldsymbol{k}} \equiv \lim _{l \rightarrow \infty} \varepsilon_{\boldsymbol{k}}(l)-\mu$ whose spectral weight $\left|\tilde{\mathcal{P}}_{\boldsymbol{k}}\right| \leq 1$. The remaining amount of spectral weight is distributed among the background which is made of some damped quasiparticles induced from the exchange interaction between fermions and bosons. We will further refer to such states as the incoherent background.

For here discussed context it is important what happens with the spectral function with respect to varying temperature. From previous studies [15] we know that the quasiparticle dispersion $\tilde{\varepsilon}_{\boldsymbol{k}}$ shows a tendency to a discontinuity around the Fermi momentum $\boldsymbol{k}_{\mathrm{F}}$ when temperatures are smaller than some value $T_{\mathrm{p}}$. This tendency effectively reflects a partial depletion of the single particle states near the Fermi energy (pseudogap). In addition to the pseudogap there appear also the other concomitant effects. Certain part of the spectral weight $\left|\tilde{\mathcal{P}}_{\boldsymbol{k}}\right|^{2}$ becomes gradually transfered to the incoherent background and, moreover, a piece of this background gives rise to the Bogoliubov shadow band [16]. It emerges in a form of the broadened peak centered at energy $\omega=-\tilde{\varepsilon}_{\boldsymbol{k}}$ as shown in Fig. 2.

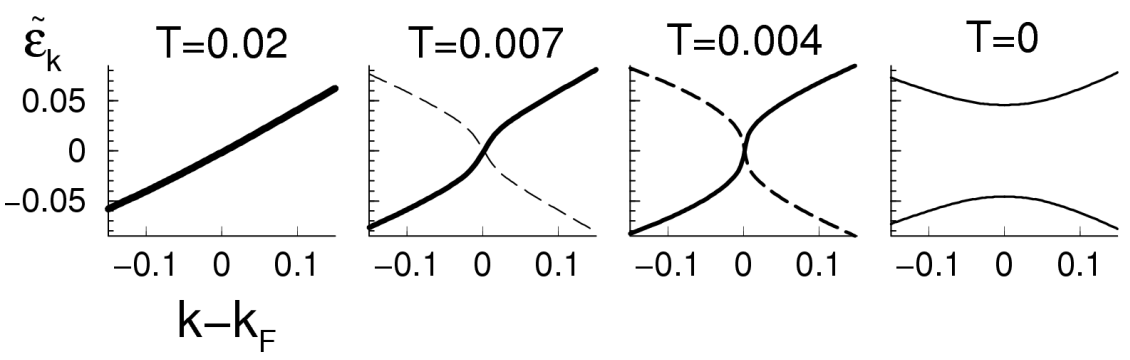

Fig. 2. Emergence of the Bogoliubov quasiparticle features in the normal state $(T=0.02,0.007,0.004)$ compared with the superconducting ground state $(T=0)$.

We can account for appearance of the shadow band on analytical grounds. For lowering temperature bosons gather more and more at low momenta and since majority of bosons occupy the states below a certain momentum cut-off $\Lambda$ (from our estimation it is of the order of the inverse lattice spacing) we can rewrite the 
incoherent part of spectral function as

$$
\begin{gathered}
A_{\mathrm{inc}}^{\mathrm{F}}(\boldsymbol{k}, \omega) \simeq\left|\tilde{r}_{\boldsymbol{k}, \boldsymbol{q} \simeq \mathbf{0}}\right|^{2} \sum_{|\boldsymbol{q}| \leq \Lambda} \frac{n_{\boldsymbol{q}}^{\mathrm{B}}}{N} \delta\left(\omega+\tilde{\varepsilon}_{-\boldsymbol{k}}-\tilde{E}_{\boldsymbol{q}}\right) \\
+\frac{1}{N} \sum_{\boldsymbol{q} \neq \mathbf{0}} n_{\boldsymbol{q}-\boldsymbol{k} \downarrow}^{\mathrm{F}}\left|\tilde{r}_{\boldsymbol{k}, \boldsymbol{q}}\right|^{2} \delta\left(\omega+\tilde{\varepsilon}_{\boldsymbol{q}-\boldsymbol{k}}-\tilde{E}_{\boldsymbol{q}}\right) .
\end{gathered}
$$

The second part in (3) is rather weakly dependent on temperature and can be regarded as a some rigid background $A_{\text {inc,rig }}^{\mathrm{F}}(\boldsymbol{k}, \omega)$. Integrating boson states over the small momenta one obtains

$$
A_{\text {inc }}^{\mathrm{F}}(\boldsymbol{k}, \omega) \simeq \frac{n^{\mathrm{B}}}{\pi} \frac{\Gamma_{\boldsymbol{k}}}{\left(\omega+\tilde{\varepsilon}_{-\boldsymbol{k}}\right)^{2}+\Gamma_{\boldsymbol{k}}^{2}}+A_{\text {inc,rig }}^{\mathrm{F}}(\boldsymbol{k}, \omega),
$$

where $n^{\mathrm{B}}$ stands for total concentration of bosons. For $T \longrightarrow T_{\mathrm{c}}$ the Lorentzian width $\Gamma_{\boldsymbol{k}}$ shrinks to zero and therefore below $T_{\mathrm{c}}$ there appear two branches of the long-lived excitations as in the standard BCS theory.

Similar behavior was previously found within the negative $U$ Hubbard model using the quantum Monte Carlo simulations [17]. Bogoliubov-type branches were observed there in the intermediate regime of the attraction potential $U$. Also in a number of other theoretical studies [18] authors applied the BCS-like substitution for the fermion self-energy $\Sigma(\boldsymbol{k}, \omega)=\Delta_{\mathrm{pg}}^{2} /\left(\omega+\varepsilon_{\boldsymbol{k}}-\mu\right)$ which produces the same Bogoliubov-like branches in the spectrum. As far as the experimental data are concerned there is no evidence for such feature in the HTS materials. It might be however caused by too poor resolution of the present ARPES measurements [19].

\section{Spectrum of the fermion pairs}

The same procedure applied for computation of the fermion pair Green function $N^{-2} \sum_{\boldsymbol{k}, \boldsymbol{p}}\left\langle\left\langle c_{\boldsymbol{k} \downarrow}(\tau) c_{\boldsymbol{q}-\boldsymbol{k} \uparrow}(\tau) ; c_{\boldsymbol{q}-\boldsymbol{p} \uparrow}^{\dagger} c_{\boldsymbol{p} \downarrow}^{\dagger}\right\rangle\right\rangle$ gives the following overall structure of the spectral function:

$$
\begin{aligned}
& A^{\text {pair }}(\boldsymbol{q}, \omega) \\
& \quad=\left|\tilde{\mathcal{N}}_{\boldsymbol{q}}\right|^{2} \delta\left(\omega-\tilde{E}_{\boldsymbol{q}}\right)+\frac{1}{N} \sum_{\boldsymbol{k}} f_{\boldsymbol{k}, \boldsymbol{q}-\boldsymbol{k}}\left|\tilde{\mathcal{M}}_{\boldsymbol{q}, \boldsymbol{k}}\right|^{2} \delta\left(\omega-\tilde{\varepsilon}_{\boldsymbol{k}}-\tilde{\varepsilon}_{\boldsymbol{q}-\boldsymbol{k}}\right),
\end{aligned}
$$

where $f_{\boldsymbol{k}, \boldsymbol{p}}=1-n_{\boldsymbol{k} \downarrow}^{\mathrm{F}}-n_{\boldsymbol{p} \uparrow}^{\mathrm{F}}$ and the meaning of coefficients $\tilde{\mathcal{N}}_{\boldsymbol{q}}, \tilde{\mathcal{M}}_{\boldsymbol{q}, \boldsymbol{k}}$ is explained in Ref. [20]. The first term of (5) describes the long-lived quasiparticles with the renormalized energy $\tilde{E}_{\boldsymbol{q}} \equiv \lim _{l \rightarrow \infty} E_{\boldsymbol{q}}(l)-2 \mu$, while the second one corresponds to the incoherent background formed over the region determined by renormalized fermion energies $\tilde{\varepsilon}_{\boldsymbol{k}}$.

The single particle fermion excitations $\tilde{\varepsilon}_{\boldsymbol{k}}$ are gaped near the Fermi energy thereof the incoherent part is then expelled from low lying energies. Yet, some amount of the incoherent pair spectrum survives because the fermionic states are 
only partly depleted [15]. Appearance of the pseudogap helps thus to emerge the long-lived fermion pairs out of the incoherent background. This effect allows for observation of the short-time correlations between fermion pairs because their finite lifetime gradually increases for $T \rightarrow T_{\mathrm{c}}$.

At transition temperature the static pair susceptibility diverges in the long wavelength limit $\boldsymbol{q}=\mathbf{0}$ and consequently fermion pairs become good, long-lived quasiparticles. Their propagation is no longer endangered by the incoherent states because they are pushed to energies $\omega \geq 2 \Delta_{\mathrm{sc}}$. The broken symmetry is also additionally characterized by appearance of the collective (Goldstone) sound-wave branch $E_{\boldsymbol{q}}=c_{1}|\boldsymbol{q}|$ [21]. From our approximate treatment of the flow equations for 3-dimensional case of the BF model we found evidence that the collective mode arises already above $T_{\mathrm{c}}$. At low momenta it is surrounded by the incoherent background states therefore the long-lived fermion pairs exist only for sufficiently large momenta $q>q_{\text {crit }}(T)$. Hence such finite momentum fermion pairs split off from the incoherent background and become long-lived. The pair coherence is thus possible to establish in the normal state on a certain restricted spatial distance $\delta x<h / q_{\text {crit }}(T)$. It gradually increases upon approaching $T_{\mathrm{c}}$. We conjecture that such short-range correlations were probably responsible for the unusually large Nernst coefficient above $T_{\mathrm{c}}[4]$.

\section{Conclusions}

We studied here the precursor features of superconducting order which might appear in the normal state of HTS cuprates. Strong quantum fluctuations of the order parameter are there especially pronounced in the underdoped to optimally doped samples because the wave functions of preformed pairs do weakly overlap with one another.

Upon approaching the transition temperature there appear the following precursor effects: the pseudogap, various signatures of the new (Bogoliubov-type) quasiparticles and also the many-body correlations which extend over a finite (time and spatial) range. The long-lived branch of the pair spectrum splits off from a remaining incoherent background for sufficiently large momentum $q>q_{\text {crit }}$. This critical value $q_{\text {crit }}$ shifts to lower and lower momenta when temperature approaches $T_{\mathrm{c}}$. Moreover, there shows up the linear in $q$ dispersion with an almost temperature independent first sound velocity $c_{1}$ which is typical of the strongly interacting boson systems. In a close vicinity of $q=0$ this mode is damped. We claim that the above mentioned effects had crucial importance for the observed transient Meissner effect [3] and for the large Nernst coefficient [4] signifying the short-range pair correlations above $T_{\mathrm{c}}$. 


\section{Acknowledgment}

This work was supported by the State Committee for Scientific Research under the grant No. 2P03B06225. Author kindly acknowledges scientific collaboration with J. Ranninger and helpful discussions with K.I. Wysokiński.

\section{References}

[1] For a detailed discussion of the pseudogap properties we recommend the following review articles: T. Timusk, B. Statt, Rep. Prog. Phys. 62, 61 (1999); E.W. Carlson, V.J. Emery, S.A. Kivelson, D. Orgad, cond-mat/0206217; M.R. Norman, C. Pepin, cond-mat/0302347.

[2] K. McElroy, D.-H. Lee, J.E. Hoffman, K.M. Lang, J. Lee, E.W. Hudson, H. Eisaki, S. Uchida, J.C. Davis, cond-mat/0406491.

[3] J. Corson, R. Mallozzi, J. Orenstein, J.N. Eckstein, I. Bozovic, Nature 398, 221 (1999).

[4] Z.A. Xu, N.P. Ong, Y. Wang, T. Takeshita, S. Uchida, Nature 406, 486 (2000).

[5] Y.J. Uemura, Solid State Commun. 126, 23 (2003).

[6] L.G. Aslamazov, A.I. Larkin, Phys. Lett. A 26, 238 (1968).

[7] A. Larkin, A. Varlamov, cond-mat/0109177.

[8] R.E. Glover, Phys. Lett. A 25, 544 (1967); M. Strongin, O.F. Kammerer, J. Crow, R.S. Thompson, H.L. Fine, Phys. Rev. Lett. 20, 922 (1968).

[9] J.P. Gollub, M.R. Beasley, M. Tinkham, Phys. Rev. Lett. 25, 1646 (1970).

[10] R.W. Cohen, B. Abeles, C.R. Fuselier, Phys. Rev. Lett. 23, 377 (1969).

[11] J. Ranninger, S. Robaszkiewicz, Physica B 135, 468 (1985).

[12] R. Micnas, J. Ranninger, S. Robaszkiewicz, Rev. Mod. Phys. 62, 113 (1990); S. Robaszkiewicz, R. Micnas, J. Ranninger, Phys. Rev. B 36, 180 (1987).

[13] J. Ranninger, J.-M. Robin, M. Eschrig, Phys. Rev. Lett. 74, 4027 (1995); A. Romano, J. Ranninger, Phys. Rev. B 62, 4066 (2000); P. Devillard, J. Ranninger, Phys. Rev. Lett. 84, 5200 (2000).

[14] F. Wegner, Ann. Phys. (Leipzig) 3, 77 (1994); Phys. Rep. 348, 77 (2001).

[15] T. Domański, J. Ranninger, Phys. Rev. B 63, 134505 (2001); Physica C 387, 77 (2003).

[16] T. Domański, J. Ranninger, Phys. Rev. Lett. 91, 255301 (2003).

[17] J.M. Singer, M.H. Pedersen, T. Schneider, H. Beck, H.-G. Matuttis, Phys. Rev. $B$ 54, 1286 (1996).

[18] Y.M. Vilk, A.-M.S. Tremblay, J. Phys. I (France) 7, 1309 (1997); O. Tchernyshyov, Phys. Rev. B 56, 3372 (1997); Q. Chen, K. Levin, J. Kosztin, Phys. Rev. B 63, 184519 (2001).

[19] T. Eckl, W. Hanke, S.V. Borisenko, A.A. Kordyuk, T. Kim, A. Koitzsch, M. Knupfer, J. Fink, Phys. Rev. B 70, 094522 (2004).

[20] T. Domański, J. Ranninger, Phys. Rev. B, 2004, accepted for publication.

[21] T. Kostyrko, J. Ranninger, Phys. Rev. B 54, 13105 (1996). 